\title{
A Research Based on the Mode Mobile Terminals' of Digital Library Application
}

\author{
Xin $Z \mathrm{HANG}^{\mathrm{a}}$, Dingli SONG ${ }^{\mathrm{b}}$, Jianxin $\mathrm{ZHOU}^{\mathrm{c}^{*}}$, Fuxing $\mathrm{YU}^{\mathrm{d}}$, Shu $\mathrm{YAN}^{\mathrm{e}}$ \\ North China University of Science and Technology, Tangshan, 063009, China \\ aemail:sdlhr1006@sohu.com, bemail: sdlhr617@sohu.com, email: zhoujianxin1977@126.com, \\ demail:yfx626@126.com, eemail:1183526779@qq.com
}

\section{Keywords: Mobile Terminal; Digital Library; Application Mode; Information Push}

\begin{abstract}
With the development of the information age, the traditional library can not meet the users' needs for information resources, it needs to be digital transformation. The mobile terminal as the currently new developed information devices, has great convenience that the user can access the Internet anytime, anywhere. Thus, the author combined with application features of intelligent mobile terminals currently, and I detailed a analysis of application mode which based on the information push of the mobile terminal digital library.
\end{abstract}

\section{Introduction}

With the advent of the information age, especially the increased scale of large data processing, vast amounts of data the user wants in the ocean difficult to pinpoint the information needed for the purpose of increasing, and users are no longer satisfied with the traditional single application mode, the pursuit of the application of diversity and richness ${ }^{[1]}$. At present and for a long period of time, mobile phone, tablet and other mobile devices based user also increasing the future, obviously, if you can use this model to obtain the information they want, it will be a choice ${ }^{[2]}$. Thus, the author of the current application features intelligent mobile terminals, based on a detailed analysis of the application mode mobile terminals push digital library, hoping to provide a reference for similar practices $^{[3]}$.

\section{Overview of mobile terminals and digital library}

The features of Mobile terminals to carry out library service. We often say that mobile terminals including mobile phones, tablet PCs and notebook computers and others that can be used on the move devices, now used widely mainly is smart phones and tablets ${ }^{[4]}$. Using the mobile terminal carried out Library services has a series of unique features:

Portability. The mobile terminal tend to portable, you can carry, can receive or access library resources from anywhere. Compared to traditional desktop access, it with great convenience.

Efficient. Users do not need to go to the library to search literature resources through the library desk, after retrieving even waste time looking for, then they can locate the target document resources they need. The users can search options through the digital library of the mobile terminal can be positioned literature they need within seconds, it greatly improving efficiency.

Instantaneity. Users do not need to set aside time to go to the library, not to worry about opening and closing time or holidays. As long as the user's have free time, they can access digital library through their mobile devices, even twenty-four-hour online they can be access to information resources what they want.

Economical efficiency. The birth of the digital library, which greatly reduces the cost of the library, reflecting the superior economical efficiency. For example, the traditional library is expensive, it requires a lot of manpower in the literature storage and maintenance. But the Digital Library of hundreds of thousands of words often only need a few megabytes of space, a large

\footnotetext{
* Corresponding author: Jianxin ZHOU, associate professor, College of Electrical Engineering, North China University of Science and Technology, Email:zhoujianxin1977@126.com, Tel:15032902429.
} 
savings in costs.

Scalability. With the growing popularity of mobile terminals, allowing more people to become library users. At the same time because of the time and place that limiting users rarely enter the library, they can also enjoy library service very convenient by virtue of their mobile devices.

The basic characteristics of the digital library. As the name implies, the digital library is a database which is a large collection of digital materials, including digital form of library resources nowadays, new purchasing packaged electronic publications, full-text database, e-publications and other free resources purchase or download over the Internet and related services to provide remote information retrieval system ${ }^{[5]}$. Which has a series of basic characteristics:

The way to Digital storage. Document books are the most important support information in the library and digital library is no exception, It is only stored in the form of digital books and resources used literature knowledge. Digital libraries and traditional libraries have a big difference on the storage medium, the content stored in the digital library can exist in many forms, such as text, sound, images, animation and three-dimensional shape and the like. And digital libraries stored in multiple formats to document format, for example, can be stored in TXT, PDF, DOC, etc., for example with pictures, it can be stored in GIF, PNG and JPEG and other forms. Under the circumstances, the digitized resources are mainly digital discs, machine-readable database and online digital information, including a literature and secondary literature and tertiary source.

The Intelligent way to Retrieval. Digital Library abandon the problems in the difficult traditional retrieval design of a unified search interface, greatly enhance the intelligence of retrieval. And it also can design personalized proactive approach based on the needs of users, users do not need to study or learn methods and techniques of various databases before retrieval. Of course, the search is based on artificial intelligence, the user can use their familiar language, constantly optimize the keywords, to narrow the search target. Retrieving object on the display to be constructed by a variety of ways.

The service concept to "User-centric". The purpose of the development of digital libraries is to make users access and use of the information they need highly flexible. Therefore, the concept of digital library services are user-centric. Users through the extensive user interface, a good search function, intuitive interpersonal interface provided by the digital library, anytime, anywhere digital library bearing according to user needs an organization's information resources.

Inevitability of personalized service development. The personalized service, refers to digital libraries should according to the user's basic information, including background knowledge hierarchy of needs and behavior as the basis to build targeted user model user-centric, proactive matches, the user hoof election information resource demand, delivered to the user's terminal through personalized service system. Early digital library collection resources in digital technology as the core, and then be distributed digital information resources interoperability and integration-centric, and now is the user's information needs and core. The focus of the business model of digital library development from the traditional focus on library collection resources to focus on technology to focusing on the customer. The digital library is now in the collection resource-based, technology-supported, personalized service-oriented services. Personalized service will be the only way for the development of digital libraries ${ }^{[6]}$.

The personalized service of digital library has the following advantages: (1) continue to meet the growing customer demand for information services; (2) has greatly improved the efficiency of information resources. And compared to traditional lending literature resources, digital resources has the characteristics of reused many times, it will greatly improve the efficiency of information resources; (3) to enhance the quality of library service effectively. Because digital library integrated a wide range of intelligent features that make more convenient to access information resources, and also can make use of digital libraries to enhance communication between users and libraries, so as to enhance the quality of library services.

Therefore, if library want to better survival and development in the future, personalized service will be the required development direction. Nowadays, with the rapid development of Internet and mobile Internet, it is bring an excellent opportunities for personalized development of digital 
libraries. So, it is necessary to create a personalized service through mode service to library users.

\section{A research based on the mode mobile terminals' information push of digital library}

In the above, this paper analysis the necessity about personalized services based on the mobile terminal of digital library. I believe that can combine with personalized service, to launch application mode with information push service, service to customers, that improve the quality of library services.

Process in Information Push Service. Who used the Internet, who will come into contact with the model-information push service. For example, when we use Tencent QQ chat software, Tencent news mini-window will pop up, it will from time to time for the user to send a daily news user attention, which is called the information push. The main content of this model is that users do not need to take the initiative to search for information, and the information will automatically "delivered to their doorsteps." Currently, there are an APP called "headlines today", he successfully used this model, gathering a large number of users, won commercial success ${ }^{[7]}$.

In the digital library, this information push personalized service, used to track behavior, analyzing the user's personality habits, extract the user's needs, then allowing memory resources based on the information needs of screening, matching, processing, sorting, and then the final results by way of vertebral customized to the user ${ }^{[8]}$. The entire process mainly including four aspects there are login Register of user, to build a user model, screening and matching resources, user feedback suggestions. Procedures in detail are as follows in Fig.1:

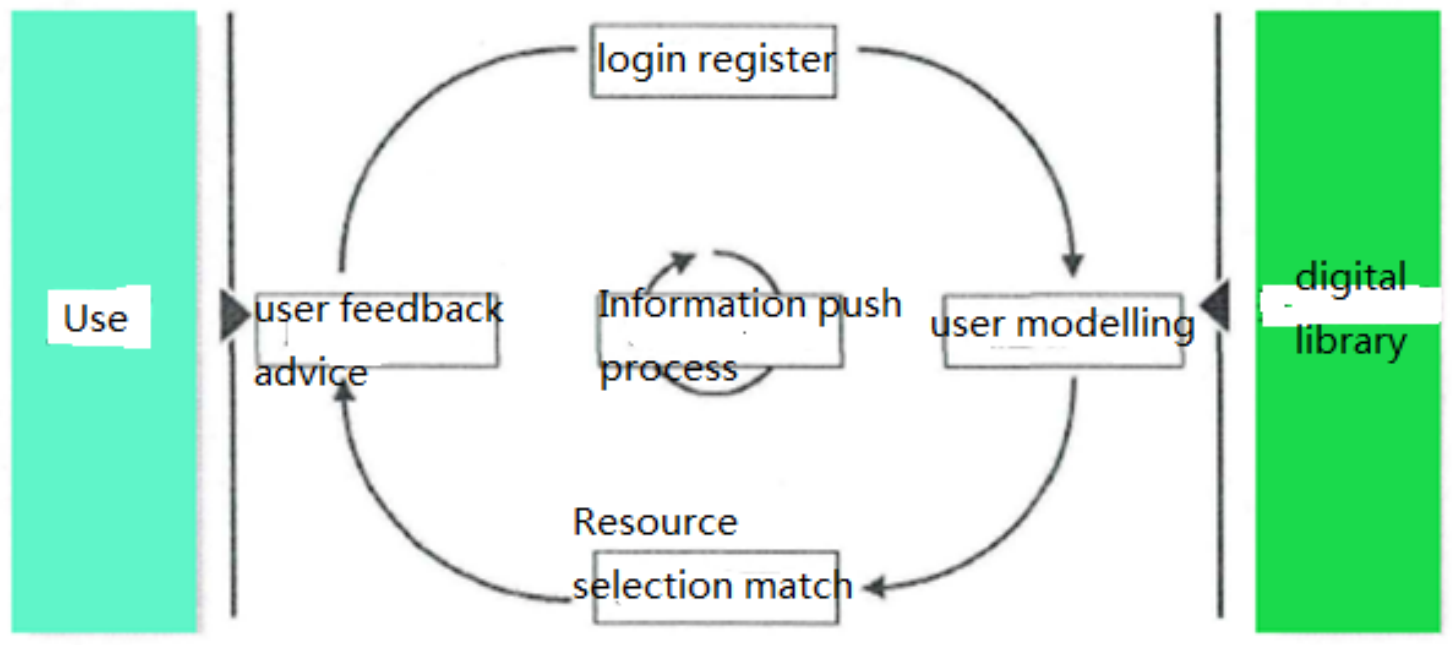

Figure 1 Procedures in detail

Information push method of Digital Library. Based on the current usage of a mobile terminal, information push way of digital libraries can be used in three modes: client, WAP and SMS services web services and so on.

Push information of client program. Client program is a software product installed on smart phones and other mobile terminals, it same as a software program on a computer, usually as a style icon displayed on the mobile terminal screen, but behind the icon is an application of a set of library services and functions in one. The user can download, install and remove by themselves. Outside the user through the client program can obtain a mobile phone in addition to text messages and WAP pages to provide all the information, but also browse the full text of digital literature resources in order to achieve seamless connection with the library. In short, the client program is to use the library from the line moved online, it will be a library of all the services and functions are digitized.

WAP Push information of Web Services. WAP is an abbreviation for Wireless Application Protocol, an implementation of application protocol standard combination of mobile phone and Internet, WAP is a global unified and open standards. Through WAP technology, users can access on mobile phones and other mobile terminal network a wealth of information resources, but also can enjoy a range of services available on the network. WAP Web Services users can not only get all the services SMS push services can also be conducted online bibliography query, view popular books 
ranking and retrieval of electronic resources and other operations. However, due to immature technology, the vast majority of digital library services through WAP pages do not provide full-text reading services, users can only access to electronic documents basic information.

Push information service of SMS. SMS messages can be the information automatically generated by the library personalized service system, and also be formed under the user customize customized for presentation to a user profile library, library services, personal information verification, time to remind and others corresponding function. Mobile phone short message service for less demanding user terminal equipment, it is widely covered by the range of users. However, because SMS is very limited in the number of words on the push and resource format can only be used to push such expiration reminder, extended Recall, briefing books and some of the notification classes, is a relatively rudimentary information push services.

Improvement of Digital Library. According to the current situation, while many of our library does not use these three ways of information push, mostly in the form of a library only mobile phone short messages and WAP pages, which limits the digital books to some extent Museum of development, needs to be improved. Meanwhile, the digital library but also to take measures in the range of users, content diversity.

First, the digital library should intensify efforts to develop mobile client, the good move client not only can increases the real-time in the interaction, and can significantly enhance the performance of digital libraries and reduce costs. Secondly, we must build a user-centric service philosophy, the personalized service of digital library should to the user's needs as the center, to enhance customer satisfaction. Third, we must strive to expand the user's range to the University Library, for example, their clients may not be limited to students and faculty, the best you can to the level of social service. Fourth, we must strive to build a distinctive information resources and services. Since the library has a large collection of resources, these characteristics are built on the basis of information resources and services to provide unique services for a particular user.

\section{Conclusion}

In summary, with the development of Internet in China, China's Internet users surfing habits are changing, with the mobile terminal as the representative of the way it is gradually being affected by favor, which makes digital library become an inevitable development of traditional library trend. Therefore, we believe that only the actual needs of users as the center, designed to develop closer to the actual personalized service, in order to make the library continues to adapt to social development in order to bring a better experience.

\section{Acknowledgements}

The author would like to thank the anonymous reviewers for their constructive and insightful comments for further improving the quality of this work. This work was supported by the Key Projects of Hebei Provincial Education Department (No. ZD2015059).

\section{References}

[1] Jia Hong. The research on personalized service of digital library [J]. Modern Information, 2006, (3): 71-73.

[2] He Wei, Cao Jindan, Diao Yunmei. The application of mobile equipment in libraries' patrons service [J]. Information Science, 2006, (5): 767-771.

[3] Ouyang Feng. Web data mining and academic digital library personalized service [J]. Modern Information, 2008, (1): 103-104.

[4] Liu Xia. The application of mobile terinals in the library [J]. Sci-tech Information Development \& Economy, 2008, (12): 11-13. 
[5] Huang Weiyu. The research on personalized service of digital library [J]. Modern Information, 2007, (10): 81-83.

[6] Sun Ping. Research of library service technology based on mobile terminal [J]. Modern Information, 2007, (12): 133-135.

[7] Li Huiqin. The digital library service model base on cloud computing [J]. Science and Techonlogy Innovation Herald, 2010, (31): 209-210.

[8] Li Yanguo. The applicaton of the intelligent library based on the Android mobile terminal [J]. Sci-tech Information Development \& Economy, 2012, (15): 23-25. 